\title{
Large Granular Lymphocytes with Tortuous and Twisted Shapes of Aggressive Natural-Killer Cell Leukemia
}

Hui Zhu and Chunrui Li*

Department of Hematology, Tongji Hospital of Tongji

Medical College, Huazhong University of Science and

Technology, China

*Correspondling author: Chunrui Li, Department of Hematology, Tongji Hospital of Tongji Medical College, Huazhong University of Scienceand Technology, China

Received: September 20, 2018; Accepted: October 23, 2018; Published: October 30, 2018

\section{Clinical Image}

A 50-year-old man presented with a 3-week history off ever, nose bleeding, lymphadenopathy, hepatomegaly, and splenomegaly. Blood tests revealed a hemoglobin concentration of $98 \mathrm{~g} / \mathrm{L}$, a platelet count of $116 \times 10^{9} / \mathrm{L}$. White blood cell count was $3.73 \times 10^{9} / \mathrm{L}$, with $7 \%$ large granular lymphocytes. The serum lactate dehydrogenase was markedly elevated (554U/L). Cytogenetic analyses show eda highly complex karyotype (Figure 1). The FDGPET/CT demonstrated hepatomegaly and splenomegaly with normal tracer uptake.

The Peripheral Blood (PB) and Bone Marrow (BM) smears show ed infiltration of Large Granular Lymphocyte (LGLs). The morphology of the LGLs show ed tortuous and twisted shapes with basophilic cytoplasm, coarse Europhilic granules. The nuclei showed a slightly immature chromatin pattern and in conspicuous nucleoli $(A, B)$. The infiltration of LGLs in the BM biopsy tissue shows the

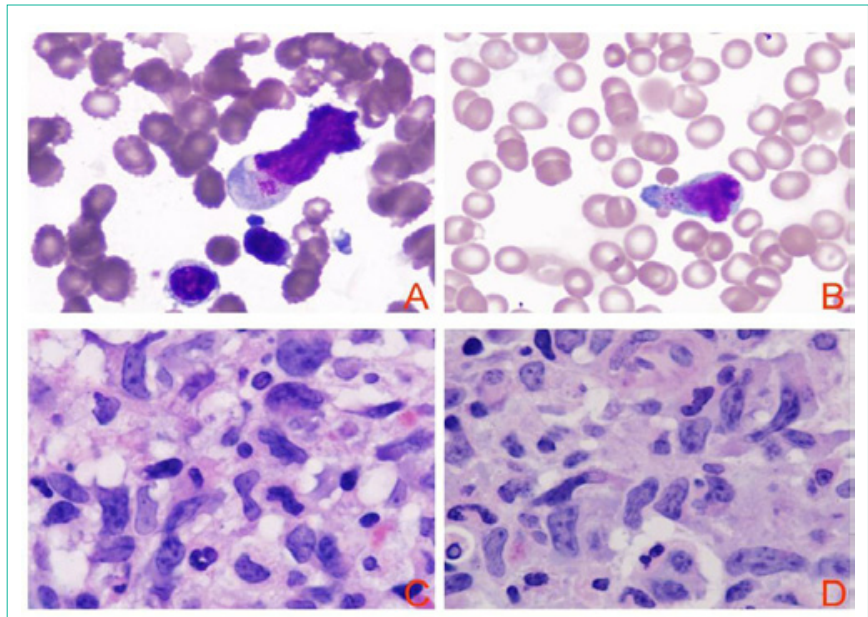

Figure 1: Cytogenetic analyses show eda highly complex karyotype.

extensive involvement of large-size lymphoid cells with irregular nuclear contours and distinct nucleoli (C,D). Immunophenotype of the LGLs by flow cytometric analyses were typically positive for CD56, CD2, CD7, and CD16, and were negative for CD3, CD4, CD5, $\mathrm{CD} 8$, and CD57. A diagnosis of aggressive NK-cell leukemia was made. The patient received intensive chemotherapy (L-asparaginase, high-dose of methotrexate and dexamethasone regimens) but died of neutropenic fungal infection. 